RUNNING HEAD: RETHINKING ONCE-WEEKLY THERAPY

September 18, 2020; ARTICLE ACCEPTED FOR PUBLICATION, Administration and Policy in Mental Health and Mental Health Services Research.

Retiring, Rethinking, and Reconstructing the Norm of Once-Weekly Psychotherapy

Jessica L. Schleider, Ph.D., ${ }^{\text {* }}$ Mallory L. Dobias, B.S., ${ }^{\text {a }}$

Michael C. Mullarkey, Ph.D., ${ }^{a} \&$ Thomas Ollendick, Ph.D. ${ }^{\text {b }}$

${ }^{a}$ Department of Psychology, Stony Brook University

${ }^{\mathrm{b}}$ Department of Psychology, Virginia Polytechnic Institute and State University

*Corresponding author and primary contact for public and scientific inquiries about this manuscript. Tel:

631632 4131; jessica.schleider@stonybrook.edu; Stony Brook University, Stony Brook, NY, USA,

11794- 2500

Disclosures. JLS receives grant and research support from the National Institutes of Health

(DP5OD28123), the Klingenstein Third Generation Foundation, the American Psychological Foundation, the National Science Foundation, and Limbix, Inc. MLD receives research funding from the Psi Chi Honor Society. JLS, MLD, and MCM have co-written a forthcoming therapeutic workbook for adolescents, to be published by New Harbinger Publications. JLS is under contract with Oxford University Press to co-edit a book on low-intensity youth mental health interventions. JLS serves on the scientific advisory board for Walden Wise. The authors report no other financial conflicts.

\title{
CITATION
}

Schleider, J. L., Dobias, M. L., Mullarkey, M. C., Ollendick, T. (2020, in press). Retiring, Rethinking, and Reconstructing the Norm of Once-Weekly Psychotherapy. Administration and Policy in Mental Health and Mental Health Services Research. 


\section{Retiring, Rethinking, and Reconstructing the Norm of Once-Weekly Psychotherapy}

Evidence-based psychotherapies hold clear potential to alleviate mental health problems (Cuijpers et al., 2020; Weisz et al., 2017), yet there is no scientifically-driven consensus for how long treatment should last (treatment duration, including total numbers of hours or weeks a treatment might last) or how often sessions should occur (treatment frequency). In practice, once-weekly therapy is the dominant outpatient service available to youths and adults alike, largely due to long-held beliefs and insurance companies' limiting reimbursable treatment-time to 50-minute, weekly sessions. But ubiquity cannot be mistaken for clinical or practical superiority. Indeed, weekly hour-long therapy sessions are among numerous treatment structures that can help patients achieve clinical gains, with recent trials supporting the utility of brief, intensive, and concentrated treatments for widely-varying problem types (Dobias, Schleider, \& Ollendick, 2020). Further, existing psychological services-dominated by weekly, outpatient options - fall short of meeting population-level mental health needs. Most youths and adults with psychiatric disorders never access care due to financial and logistical constraints, and among those who do, premature drop-out is common (Abel et al., 2020; Burns et al., 1999; McKay \& Bannon, 2004). Among those who do access psychological support, receipt of evidence-based intervention remains rare, often taking the form of unstructured, brief interactions with physicians (e.g., in primary care settings) or healthcare workers with limited mental health training (Kazdin, 2019). Despite repeated calls to diversify treatment options, and to ensure that these diverse options are backed by scientific evidence (Kazdin, 2019; Schleider, Dobias, Sung, \& Mullarkey, 2020), the "weekly therapy hour"-in many cases, absent of any quality assessment ensuring reliance of evidence-based approaches-remains the practical default.

Given limited accessibility of, and significant dropout from, weekly outpatient therapy (suggesting that many are unable to access treatment as it is routinely provided), and the established efficacy of alternative treatment formats (suggesting that treatment may be redesigned to improve accessibility without sacrificing clinical utility), we assert that it is our field's ethical obligation to retire 
and rebuild the longstanding "default" to once-weekly outpatient services. ${ }^{1}$ To be clear, we do not endorse eliminating weekly psychotherapy as an option for patients; many once-weekly, evidence-based treatments, if delivered as intended, may benefit patients greatly. However, repositioning evidence-based weekly therapy as one of many treatment options, and improving the availability of additional, diverse evidence-based service types, may strengthen the accessibility, flexibility, and potentially the effectiveness of mental health treatment overall.

\section{Treatment need not occur once-weekly.}

Psychotherapies need not be delivered in a once-weekly format to effect clinical improvement. One intensive outpatient treatment, involving four consecutive days of exposure and response prevention treatment, has substantially reduced OCD symptoms for adults up to four years later (Hansen et al., 2019; Kvale et al., 2018) and adolescents up to six months later (Riise et al., 2016, 2018). Other trials indicate that an 8-day, 20-hour treatment involving cognitive-behavioral techniques-including interoceptive exposures and psycho-education — can reduce symptoms of specific phobia, social phobia, and GAD (Gallo et al., 2012). Written-exposure therapy, an exposure-based treatment involving recalling, processing, and making meaning of past traumatic experiences via timed sessions of expressive personal writing, has produced clinically-significant reductions in PTSD after just 3-4 hours of intervention-and is non-inferior, and carries lower dropout-rates (6\% vs. 39\%) than lengthier, evidence-based alternatives (e.g., Cognitive Processing Therapy; Thompson-Hollands et al., 2019). Clearly, cookie-cutter treatment formats are unnecessary for meaningful therapeutic change. Indeed, large-scale trials and systematic reviews of treatments for anxiety disorders, depression, and behavior problems indicate that non-weekly interventions - from self-guided supports to brief, one-session treatments to concentrated, week-long interventions - can yield benefits approximately equivalent to once-weekly evidence-based

\footnotetext{
${ }^{1}$ In the present article, we focus our discussion on outpatient mental health services, given the numerous structural and practical differences between inpatient and outpatient mental health treatment options for individuals. As such, our discussion is centered on outpatient psychotherapy options, which collectively represent the majority of mental health services that individuals receive in their lifetimes.
} 
psychotherapy, both for youths and adults (Beevers et al., 2017; Öst \& Ollendick, 2017; Schleider, Dobias, Sung, \& Mullarkey, 2020; Stoll et al., 2020; Thompson-Hollands et al., 2019).

Breaking away from traditional delivery models may even carry advantages over once-weekly options. A metaregression including 70 psychotherapy trials found session frequency — rather than number of sessions or duration of therapy — was strongly associated with treatment efficacy for depression (Cuijpers et al., 2013). Controlling for total number of sessions, treatment effect size was notably higher among twice-weekly treatments, versus once-weekly treatments (increase of $g=.45$ ). Higher session frequency in the first 3 months of treatment is associated with faster improvement and recovery (Tiemens et al., 2019). Further, patients randomized to receive twice-weekly sessions experienced greater depression reductions than those offered once-weekly therapy (Bruijniks et al., 2020). Thus, defaulting to once-weekly treatment may fail to optimize patients' clinical progress.

\section{More treatment—in hours, days, or weeks—is not always better.}

Brief, evidence-based treatments, including some lasting just a few hours or a few days, can benefit many populations and problem types. Meta-analytic evidence suggests a single session intervention (SSI) can significantly improve anxiety, conduct problems, and substance use in youth (Schleider \& Weisz, 2017), and recent trials indicate that SSIs can reduce youth depression (Schleider \& Weisz, 2018; Schleider et al., 2019). Evidence across 58 studies indicates interventions lasting <5 minutes can have similar effects on young adults' problem drinking, versus multi-session interventions (TannerSmith \& Lipsey, 2015). Single-session, walk-in therapy is associated with symptom improvement in adults ages 18-80 and can reduce clinic wait-list length (Harper-Jaques \& Foucault, 2014).

Still, even if patients can benefit from brief treatments, wouldn't more treatment be better? Some correlational work suggests receiving more sessions relates to greater symptom improvement (Hansen et al., 2002), but this evidence is mixed (King, 2015). Indeed, correlational analyses cannot establish whether more treatment causes greater improvement. RCTs where patients are randomized to receive more versus fewer sessions for specific phobia (Öst et al., 1997), panic disorder (Roberge et al., 2008), insomnia (Edinger et al., 2007), PTSD (Deblinger et al., 2011), and substance abuse (Covi et al., 2002) 
consistently find no evidence for longer treatments' clinical superiority. The assumption that "more treatment is better" is unsupported by gold-standard evidence.

\section{Brief and personalized?}

"Personalized" treatment refers to therapies that are tailored to an individual's specific presenting needs. A course of treatment may be "personalized" in multiple ways, including through matching individuals with particular therapies most likely to benefit them based on pre-treatment characteristics (e.g., symptom severity), or altering the course of treatment based on changing needs, problems, or preferences as therapy progresses. One concern around brief treatments and SSIs is that their brevity might preclude personalization to individual patients' needs. This concern is misguided for at least two reasons. First, many brief treatments are "problem-agnostic" by necessity (e.g., walk-in single session therapy): They are inherently designed to adapt to the patient's presenting problem as they understand it - not to a particular clinical diagnosis, which many therapy protocols are designed to target (HarperJaques \& Foucault, 2014; Schleider et al., 2020). Thus, many brief treatments may be easier to personalize than traditional, manualized treatments centering diagnostic-criteria.

Second, psychotherapy may be personalized not just during the treatment, but prior to treatment, as well. Indeed, many treatment personalization attempts have involved "matching" particular patients with best-fit interventions before services begin (Cohen \& DeRubeis, 2018). This personalization approach may be especially important with respect to the effectiveness and accessibility of mental health services, as patients routinely see $10+$ outpatient providers before accessing treatment they perceive as helpful (Harris et al., 2020). Given sufficiently-large samples, artificial intelligence could help identify precision treatment "rules" indicating which treatment is most likely to benefit particular patients (Bickman, 2020). Most traditional RCTs have too few participants to reliably identify precision treatment rules (Kessler et al., 2019), but online SSI research may overcome this obstacle. In the previous year, our group has recruited over 900 youths for an open-label trial of SSIs (Schleider, Dobias, Sung, Mumper, et al., 2020); 302 participants for an online RCT testing an SSI targeting parents experiencing anxiety (NLM, NCT04453865); and >500 adolescents for another online SSI RCT (NLM, NCT04498143). Large 
datasets aggregated across affordable, online SSI trials could be leveraged to develop precision-treatment "rules" with great statistical precision, and at a long-impossible rate, thus rapidly forwarding efforts to reduce psychopathology's public health burden (Kazdin, 2019).

\section{Recommendations \& Conclusions}

Clearly, diverse cost- and time-efficient treatment structures—not just once-weekly psychotherapy—can yield meaningful clinical benefits for youths and adults. Treatment utilization patterns suggest that many clients cannot consistently access or complete weekly treatment involving months-long commitments; likewise, it is unrealistic to think this model would be preferable to individuals seeking high-quality care. However, major structural changes to our mental healthcare ecosystem are needed to broaden access to and awareness of diverse treatments. We offer the following recommendations as first-steps:

Therapist Training. Therapists should be trained to deliver treatment in varying formats and structures, and once weekly therapy should be identified as just one of many evidence-based treatment approaches. This will require a major revolution in training programs in clinical psychology and allied disciplines. Indeed, it may involve overhauling how we are willing to define "treatment" for psychological distress, and acknowledging that "treatment" can occur in a wide variety of settings, with or without a trained provider present, over an hour or many months, and within or beyond brick-andmortar clinic. This expanded understanding of "treatment" may create new roles and opportunities for mental healthcare providers, including flexible utilization of widely-varying forms of therapeutic client supports both in and out of traditional "sessions." It will also require sustained commitment to questioning longstanding assumptions about when, where, and how clinical progress may be achieved.

Research Priorities. The vast majority of treatment trials focus on weekly therapy sessions. However, a robust and growing body of literature has revealed that other brief, intensive and concentrated approaches can work equally well. It will be important that we personalize these approaches and determine for whom they are most effective. 
Insurance Reimbursement. Insurance companies must provide flexible structures for supporting non-weekly psychotherapy - for instance, reimbursing by time spent in treatment regardless of frequency, intensity, or duration. It is most cost-effective to cover treatment that is efficient and that "works." There must be flexibility such that, if weekly therapy is needed for certain people — which it naturally will bethat will be coverable, too. The key is making therapy sufficiently accessible and flexible to meet all individuals' clinical needs.

Treatment Quality Assessment. Just as with any mental health treatment, it is critically important to ensure that brief treatments and SSIs are monitored for quality-including their adherence to evidencebased models and practices. Without ensuring high-quality treatment content, diversifying the structure of available interventions will fail to reduce mental illness on a large scale. Brief, digital and self-help interventions are relatively straightforward to monitor for quality, as they are often standardized by design; quality assessment of therapist-delivered brief interventions must be supported via continued promotion of measurement-based approaches to treatment across disciplines (see Bickman, 1996, \& Garland, Bickman, \& Chorpita, 2010, for further discussion on this topic).

In sum, we do not endorse abandoning weekly therapy; rather we submit that it cannot ethically remain the "norm" or the sole format for the delivery of evidence-based treatments. Evidence supports the use of brief, intensive and concentrated treatments; they can be more efficient than longer treatments, more cost-effective, and more accessible (Ollendick, Öst, \& Farrrell, 2018; Ollendick, Ryan, et al., 2018). To some extent, psychological treatment provision is already being overhauled by the COVID-19 pandemic, including rapid normalization and expansion of telehealth and digital interventions. This moment presents a prime opportunity for our field to reconsider how psychological services can and should be structured - both regarding session frequency and what a "session" looks like, and deployment of brief, concentrated therapies without sacrificing personalization potential. Doing so could create more accessible, ethical, and effective treatment options for clients. 


\section{References}

Abel, M. R., Bianco, A., Gilbert, R., \& Schleider, J. L. (2020, in press). When is Psychotherapy Brief? Considering Sociodemographic Factors, Problem Complexity, and Problem Type in U.S. Adolescents. Journal of Clinical Child and Adolescent Psychology.

Beevers, C. G., Pearson, R., Hoffman, J. S., Foulser, A. A., Shumake, J., \& Meyer, B. (2017). Effectiveness of an internet intervention (Deprexis) for depression in a united states adult sample: A parallel-group pragmatic randomized controlled trial. Journal of Consulting and Clinical Psychology, 85(4), 367-380.

Bickman, L. (2020). Improving Mental Health Services: A 50-Year Journey from Randomized Experiments to Artificial Intelligence and Precision Mental Health. Administration and Policy in Mental Health, 47(5), 795-843.

Bickman, L., \& Salzer, M. S. (Eds.). (1997). Measuring quality in mental health services [Special issue]. Evaluation Review, 21(3).

Bruijniks, S. J. E., Lemmens, L. H. J. M., Hollon, S. D., Peeters, F. P. M. L., Cuijpers, P., Arntz, A., Dingemanse, P., Willems, L., van Oppen, P., Twisk, J. W. R., van den Boogaard, M., Spijker, J., Bosmans, J., \& Huibers, M. J. H. (2020). The effects of once- versus twice-weekly sessions on psychotherapy outcomes in depressed patients. The British Journal of Psychiatry: The Journal of Mental Science, 216(4), 222-230.

Burns, B. J., Hoagwood, K., \& Mrazek, P. J. (1999). Effective treatment for mental disorders in children and adolescents. Clinical Child and Family Psychology Review, 2(4), 199-254.

Cohen, Z. D., \& DeRubeis, R. J. (2018). Treatment Selection in Depression. Annual Review of Clinical Psychology, 14, 209-236.

Covi, L., Hess, J. M., Schroeder, J. R., \& Preston, K. L. (2002). A dose response study of cognitive behavioral therapy in cocaine abusers. Journal of Substance Abuse Treatment, 23(3), 191-197.

Cuijpers, P., Huibers, M., Ebert, D. D., Koole, S. L., \& Andersson, G. (2013). How much psychotherapy is needed to treat depression? A metaregression analysis. Journal of Affective Disorders, 149(1-3), 
$1-13$.

Cuijpers, P., Karyotaki, E., Eckshtain, D., Ng, M. Y., Corteselli, K. A., Noma, H., Quero, S., \& Weisz, J. R. (2020). Psychotherapy for Depression Across Different Age Groups: A Systematic Review and Meta-analysis. JAMA Psychiatry . https://doi.org/10.1001/jamapsychiatry.2020.0164

Deblinger, E., Mannarino, A. P., Cohen, J. A., Runyon, M. K., \& Steer, R. A. (2011). Trauma-focused cognitive behavioral therapy for children: impact of the trauma narrative and treatment length. Depression and Anxiety, 28(1), 67-75.

Dobias, M. L., Schleider, J. L., \& Ollendick, T. (2020, forthcoming). Improving the efficiency and scalability of cognitive behavioral therapies. In Comprehensive Clinical Psychology (2nd ed.). Elsevier.

Edinger, J. D., Wohlgemuth, W. K., Radtke, R. A., Coffman, C. J., \& Carney, C. E. (2007). Doseresponse effects of cognitive-behavioral insomnia therapy: a randomized clinical trial. Sleep, 30(2), 203-212.

Gallo, K. P., Chan, P. T., Buzzella, B. A., Whitton, S. W., \& Pincus, D. B. (2012). The Impact of an 8Day Intensive Treatment for Adolescent Panic Disorder and Agoraphobia on Comorbid Diagnoses. In Behavior Therapy (Vol. 43, Issue 1, pp. 153-159). https://doi.org/10.1016/j.beth.2011.05.002

Garland, A. F., Bickman, L., \& Chorpita, B. F. (2010). Change what? Identifying quality improvement targets by investigating usual mental health care. Making the Real-World Ideal: Changing Practices in Children's Mental Health Services. Administration and Policy in Mental Health and Mental Health Services Research, 37(1-2), 15-26. doi:10.1007/s10488-010-0279-y

Hansen, B., Kvale, G., Hagen, K., Havnen, A., \& Öst, L.-G. (2019). The Bergen 4-day treatment for OCD: four years follow-up of concentrated ERP in a clinical mental health setting. In Cognitive Behaviour Therapy (Vol. 48, Issue 2, pp. 89-105). https://doi.org/10.1080/16506073.2018.1478447

Hansen, N. B., Lambert, M. J., \& Forman, E. M. (2002). The psychotherapy dose-response effect and its implications for treatment delivery services. Clinical Psychology: Science and Practice, 9(3), 329343. 
Harper-Jaques, S., \& Foucault, D. (2014). Walk-In Single-Session Therapy: Client Satisfaction and Clinical Outcomes. Journal of Systemic Therapies, 33(3), 29-49.

Harris, M. G., Kazdin, A. E., Chiu, W. T., Sampson, N. A., Aguilar-Gaxiola, S., Al-Hamzawi, A., Alonso, J., Altwaijri, Y., Andrade, L. H., Cardoso, G., Cía, A., Florescu, S., Gureje, O., Hu, C., Karam, E. G., Karam, G., Mneimneh, Z., Navarro-Mateu, F., Oladeji, B. D., ... WHO World Mental Health Survey Collaborators. (2020). Findings From World Mental Health Surveys of the Perceived Helpfulness of Treatment for Patients With Major Depressive Disorder. JAMA Psychiatry.

Kazdin, A. E. (2019). Annual Research Review: Expanding mental health services through novel models of intervention delivery. Journal of Child Psychology and Psychiatry, and Allied Disciplines, 60(4), $455-472$.

Kazdin, A. E., \& Blase, S. L. (2011). Interventions and Models of Their Delivery to Reduce the Burden of Mental Illness: Reply to Commentaries. Perspectives on Psychological Science: A Journal of the Association for Psychological Science, 6(5), 507-510.

Kessler, R. C., Bossarte, R. M., Luedtke, A., Zaslavsky, A. M., \& Zubizarreta, J. R. (2019). Machine learning methods for developing precision treatment rules with observational data. Behaviour Research and Therapy, 120, 103412.

King, M. (2015). Duration of psychotherapy has little association with outcome. The British Journal of Psychiatry: The Journal of Mental Science, 207(2), 93-94.

Kvale, G., Hansen, B., Björgvinsson, T., Børtveit, T., Hagen, K., Haseth, S., Kristensen, U. B., Launes, G., Ressler, K. J., Solem, S., Strand, A., van den Heuvel, O. A., \& Öst, L.-G. (2018). Successfully treating 90 patients with obsessive compulsive disorder in eight days: the Bergen 4-day treatment. BMC Psychiatry, 18(1), 323.

McKay, M. M., \& Bannon, W. M., Jr. (2004). Engaging families in child mental health services. Child and Adolescent Psychiatric Clinics of North America, 13(4), 905-921, vii.

Ollendick, T.H., Öst, L-G., \& Farrell, L. J. (2018). Innovations in the psychosocial treatment of youth with anxiety disorders: Implications for a stepped care approach. Evidence-Based Mental Health, 
$21,112-115$.

Ollendick, T. H., Ryan, S. M., Capriola-Hall, N. N., Fraire, M. G, \& Austin, K. E. (2018). Have phobias, will travel: Addressing one barrier to the delivery of an evidence-based treatment. Behavior Therapy, 49, 594-603.

Öst, L.-G., Brandberg, M., \& Alm, T. (1997). One versus five sessions of exposure in the treatment of flying phobia. Behaviour Research and Therapy, 35(11), 987-996.

Öst, L.-G., \& Ollendick, T. H. (2017). Brief, intensive and concentrated cognitive behavioral treatments for anxiety disorders in children: A systematic review and meta-analysis. Behaviour Research and Therapy, 97, 134-145.

Riise, E. N., Kvale, G., Öst, L.-G., Skjold, S. H., \& Hansen, B. (2018). Concentrated exposure and response prevention for adolescents with obsessive-compulsive disorder: A replication study. In Journal of Obsessive-Compulsive and Related Disorders (Vol. 19, pp. 15-22). https://doi.org/10.1016/j.jocrd.2018.07.002

Riise, E. N., Kvale, G., Öst, L.-G., Skjold, S. H., Hansen, H., \& Hansen, B. (2016). Concentrated exposure and response prevention for adolescents with obsessive-compulsive disorder: An effectiveness study. In Journal of Obsessive-Compulsive and Related Disorders (Vol. 11, pp. 1321). https://doi.org/10.1016/j.jocrd.2016.07.004

Roberge, P., Marchand, A., Reinharz, D., \& Savard, P. (2008). Cognitive-Behavioral Treatment for Panic Disorder With Agoraphobia A Randomized, Controlled Trial and Cost-Effectiveness Analysis. Behavior Modification, 32(3), 333-351.

Schleider, J. L., Dobias, M. L., Sung, J. Y., \& Mullarkey, M. C. (2020). Future Directions in SingleSession Youth Mental Health Interventions. Journal of Clinical Child and Adolescent Psychology, 49(2), 264-278.

Schleider, J. L., Dobias, M., Sung, J., Mumper, E., \& Mullarkey, M. C. (2020). Acceptability and Utility of an Open-Access, Online Single-Session Intervention Platform for Adolescent Mental Health. JMIR Mental Health, 7(6), e20513. 
Schleider, J. L., \& Weisz, J. R. (2017). Little Treatments, Promising Effects? Meta-Analysis of SingleSession Interventions for Youth Psychiatric Problems. Journal of the American Academy of Child and Adolescent Psychiatry, 56(2), 107-115.

Stoll, R. D., Pina, A. A., \& Schleider, J. (2020). Brief, Non-Pharmacological, Interventions for Pediatric Anxiety: Meta-Analysis and Evidence Base Status. Journal of Clinical Child and Adolescent Psychology, 49(4), 435-459.

Tanner-Smith, E. E., \& Lipsey, M. W. (2015). Brief alcohol interventions for adolescents and young adults: a systematic review and meta-analysis. Journal of Substance Abuse Treatment, 51, 1-18.

Thompson-Hollands, J., Marx, B. P., \& Sloan, D. M. (2019). Brief novel therapies for PTSD: Written Exposure Therapy. Current Treatment Options in Psychiatry, 6(2), 99-106.

Tiemens, B., Kloos, M., Spijker, J., Ingenhoven, T., Kampman, M., \& Hendriks, G.-J. (2019). Lower versus higher frequency of sessions in starting outpatient mental health care and the risk of a chronic course; a naturalistic cohort study. BMC Psychiatry, 19(1), 228.

Weisz, J. R., Kuppens, S., Ng, M. Y., Eckshtain, D., Ugueto, A. M., Vaughn-Coaxum, R., Jensen-Doss, A., Hawley, K. M., Krumholz Marchette, L. S., Chu, B. C., Weersing, V. R., \& Fordwood, S. R. (2017). What five decades of research tells us about the effects of youth psychological therapy: A multilevel meta-analysis and implications for science and practice. The American Psychologist, 72(2), 79-117. 